\title{
On Reconnection Phenomena in the Standard Nontwist Map
}

\author{
A. Wurm, A. Apte, and P.J. Morrison \\ Department of Physics and Institute for Fusion Studies \\ The University of Texas at Austin, Austin, TX 78712 USA \\ Received on 1 February, 2004
}

\begin{abstract}
Separatrix reconnection in the standard nontwist map is described, including exact methods for determining the reconnection threshold in parameter space. These methods are implemented numerically for the case of oddperiod orbit reconnection, where meanders (invariant tori that are not graphs) appear. Nested meander structure is numerically demonstrated, and the idea of meander transport is discussed.
\end{abstract}

\section{Introduction}

Here we consider the standard nontwist map (SNM) $M$, as introduced in Ref. [1]:

$$
\begin{aligned}
& x_{n+1}=x_{n}+a\left(1-y_{n+1}^{2}\right), \\
& y_{n+1}=y_{n}-b \sin \left(2 \pi x_{n}\right),
\end{aligned}
$$

where $(x, y) \in \mathbb{T} \times \mathbb{R}, a \in(0,1)$ and $b \in(-\infty, \infty)$. This map is area-preserving and violates the twist condition,

$$
\frac{\partial x_{i+1}\left(x_{i}, y_{i}\right)}{\partial y_{i}} \neq 0 \quad \forall\left(x_{i}, y_{i}\right),
$$

along a curve in phase space, called the nonmonotone curve.[2] Traditional studies of area-preserving maps dealt with twist maps, for which (2) is satisfied, but more recent research efforts have considered the nontwist case.

Nontwist maps describe many physical systems, including the magnetic field lines in toroidal plasma devices such as tokamaks (see e.g. [3, 4, 5, 6, 7]) and stellarators[8, 9]. Recently, it has been shown[10] that (2) is violated generically in area-preserving maps that have a tripling bifurcation of an elliptic fixed point. Apart from their physical importance, nontwist maps are of mathematical interest because important theorems concerning area-preserving maps assume the twist condition, e.g., the KAM and PoincareBirkhoff theorems. The SNM can serve as a model for the development of new proofs. Presently, only a few mathematical results exist for nontwist maps [11, 12, 2, 13].

Nontwist maps of the annulus exhibit interesting bifurcation phenomena: periodic orbit collision and separatrix reconnection. The former, which applies specifically to collision of periodic orbits of the same period, such as the socalled up and down periodic orbits that occur in the SNM, can be used to calculate torus destruction, [14] while the latter is a global bifurcation that changes the phase space topology in the vicinity of a central barrier that occurs in these maps. At the threshold of reconnection, the invariant manifolds of two or more distinct hyperbolic orbits with the same rotation number connect. Various aspects of this bifurcation have been studied over the years. Some early references are Refs. [14, 15, 16]. (For a more complete review of past work see Ref. [17].)

Key to the analytical and numerical exploration of the SNM is the map's invariance under symmetries. ${ }^{1}$ Of particular significance are indicator points,[18] fixed points of some of the symmetries of the SNM, given by

$$
\boldsymbol{z}_{1}^{(0,1)}=\left(\mp \frac{1}{4}, \mp \frac{b}{2}\right), \quad \text { and } \quad \boldsymbol{z}_{2}^{(0,1)}=\left(\frac{a}{2} \mp \frac{1}{4}, 0\right)
$$

The importance of these points was first recognized by Shinohara and Aizawa. In Ref. [19], they showed that a shearless invariant torus crosses the $x$-axis at the two points $z_{2}^{0}$ and $z_{2}^{1}$. This led them to devise the following criterion to determine the approximate location in the $(a, b)$-parameter space of the breakup of shearless invariant tori: For a given $(a, b)$ value, one of these points, e.g. $\boldsymbol{z}_{2}^{0}$, is iterated many times. If the $y$ value stays below a threshold, it is assumed that the shearless curve exists and the point is plotted. The boundary of the resulting diagram (see Fig. 7) displays a fractal-like structure. The analysis of Refs. [20, 14, 19] indicates that the highest peaks correspond to the break-up of shearless invariant tori with noble winding numbers. (See Refs. [20, 21] for discussion.)

In Ref. [18], Shinohara and Aizawa obtained the four points of (3) and used them to propose exact expressions for the reconnection threshold of even-period hyperbolic orbits. These indicator points were independently re-discovered by Petrisor[2] in the analysis of the reversing symmetry group[22] of nontwist standard-like area-preserving maps.

A very different exact criterion for the reconnection threshold was also proposed by Petrisor in Ref. [23]. For a class of standard-like nontwist maps it was shown that the hyperbolic points belonging to periodic orbits of the same period are at the reconnection threshold if their actions coincide. As noted in Ref. [23], for odd-period hyperbolic orbits in the SNM this criterion reduces to the action being zero at the point of reconnection.

\footnotetext{
${ }^{1}$ For a review of symmetry properties of the SNM and the definition of symmetry lines see Ref. [20].
} 

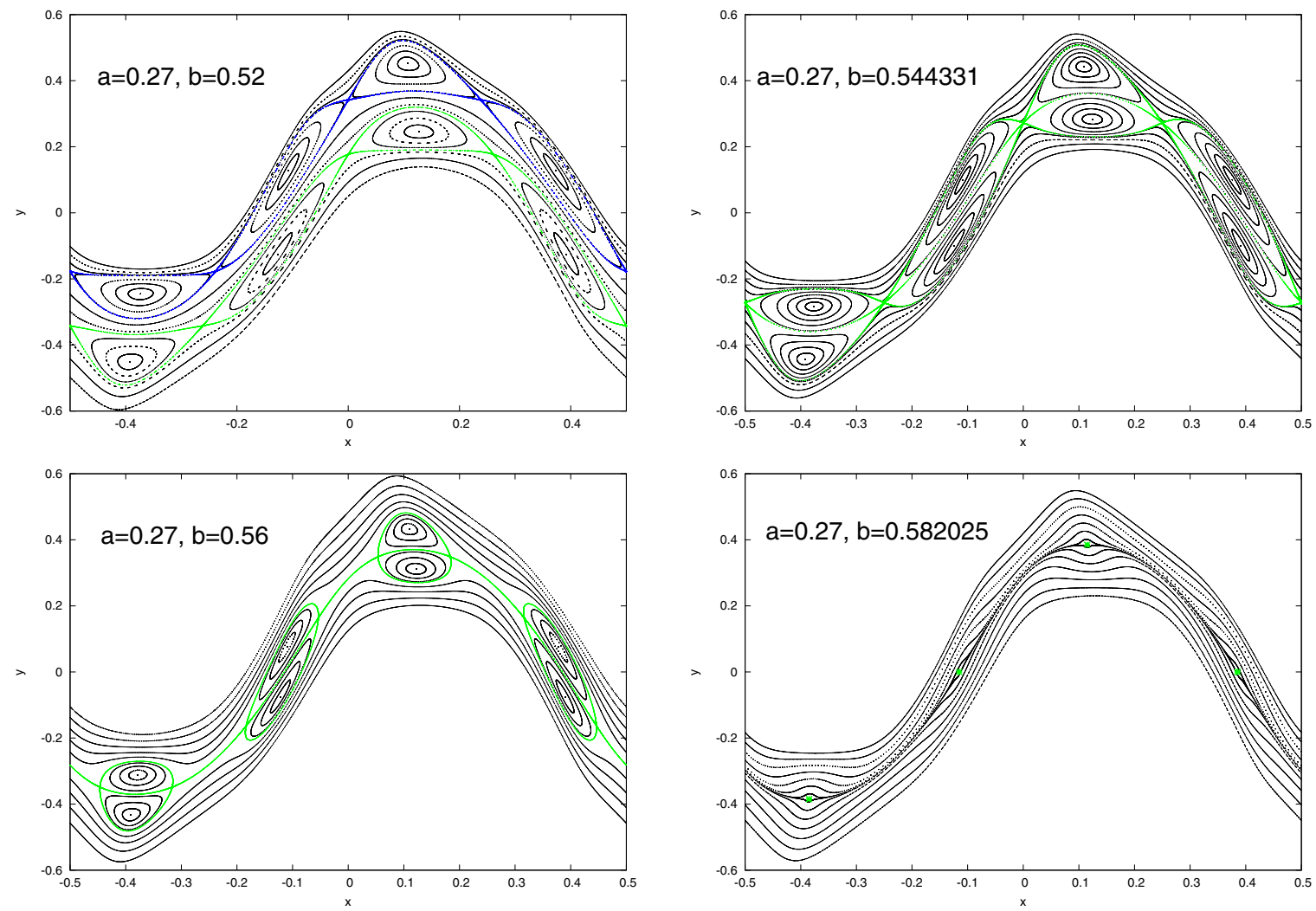

Figure 1. Depiction of periodic orbit collision for even-period orbits at $a=0.27$. The periodic orbit collision of the hyperbolic orbits on $s_{1}$ [Fig.2] is interpreted as the reconnection of the up and down island chains. The winding number of the chains shown here is $1 / 4$.

A goal of the present paper is to bring together these two exact criteria for the reconnection threshold and to implement them numerically. We aim to deepen the understanding of the behavior of the SNM in different regions in $(a, b)$-parameter space by extending the discussions of Refs. [20, 21, 14, 19, 18].

The paper is organized as follows: In Sec. 2, we describe the reconnection of hyperbolic orbits of even period, applying the method of Ref. [18] to Eq. (1). A description of the reconnection of hyperbolic orbits with odd period is presented in Sec. 3, where we also numerically implement the exact criterion of Ref. [23]. In addition, we discuss the occurrence of meanders and introduce the notion of meander transport. Section IV contains our conclusions and some directions of future research.

\section{Reconnection of even-period perio- dic orbits}

The reconnection process for orbits with even-period winding number, for which the up and down orbits on a symmetry line have the same stability type, is illustrated by the example of 1/4-orbits. As the perturbation is increased (by increasing $b$ at a fixed value of $a$ ), the hyperbolic orbits on the $s_{3}$ and $s_{4}$ symmetry lines ${ }^{2}$ collide [Fig. 2]. The phase space topology after the collision of hyperbolic orbits shows the "dipoles" of elliptic orbits [Fig. 2]. As the perturbation is increased further, the elliptic orbits collide as well, as shown in Fig. 2. No 1/4-period orbits exist for larger values of $b$. This process takes place for any two chains of periodic orbits which have the same stability type on a symmetry line.

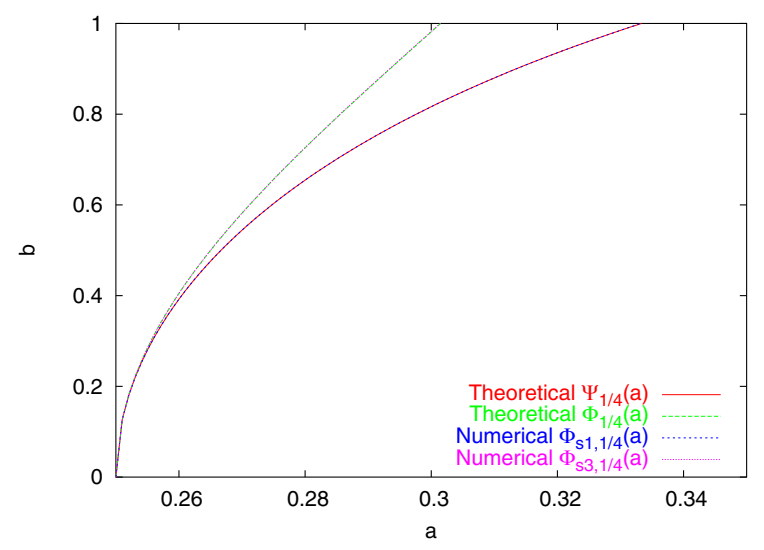

Figure 2. Comparison of the numerical bifurcation curves $(b=$ $\Phi(a)$ ) with the analytical expressions obtained using indicator points.

\footnotetext{
${ }^{2}$ See [14] for discussion of symmetry lines.
} 
The threshold for reconnection of even-period orbits, which is the same as the point of collision of their hyperbolic points, can be obtained by using the numerical observation[18] that at the point of bifurcation two of the indicator points belong to the periodic orbit. ${ }^{3}$ This implies that $M^{2 n} \boldsymbol{z}_{i}^{(0,1)}=\boldsymbol{z}_{i}^{(0,1)}$ for either $i=1$ or $i=2$, where $\boldsymbol{z}_{i}^{(0,1)}$ are the indicator points given in Eq. (3).

The symmetries of the map further imply that the indicator points map onto each other after $n$ iterations, i.e. $M^{n} \boldsymbol{z}_{i}^{(0,1)}=\boldsymbol{z}_{i}^{(1,0)}$. By solving these two equations for the two unknowns $(a, b)$, we can obtain an exact expression for the bifurcation threshold. Note that the result obtained using $z_{1}^{(0,1)}$ is the reconnection threshold $[b=\Psi(a)]$, while that using $\boldsymbol{z}_{1}^{(0,1)}$ is the collision threshold $[b=\Phi(a)]$. Fig. 2 shows that the curves obtained analytically using this method compare very well with the numerically obtained bifurcation curves (where up and down periodic orbits have collided) using the methods of Ref. [20].

\section{Reconnection of odd-period perio- dic orbits}

The reconnection scenario for the odd-period orbits is very different because the stability types of the up and down periodic orbits on a symmetry line are different [Fig. 3]. In this case, as the chains approach each other with increasing perturbation, the hyperbolic manifolds of the up and down orbits connect, as shown in Fig. 3. Further, increase of the perturbation leaves each hyperbolic orbit with a homoclinic and a heteroclinic manifold. In the region between the two chains, new periodic orbits and non-KAM tori appear [Fig. 3], i.e., orbits and tori that do not exist at zero perturbation. These tori are not graphs over the $x$-axis and have been called meanders or meandering curves. [24, 13] Such invariant tori can occur only in nontwist maps (to be more precise, in the nontwist region of the phase space). It is known that any invariant torus for a twist map must be a graph over $x$.

As the perturbation is increased further, the periodic orbits collide [Fig. 3], and are survived by the meandering tori. This reconnection process, in a related plasma physics context, was conjectured by Stix in [6, page 523]. He stated (emphasis and figure references are ours):

Looking now at the nonlinear growth of the double-tearing mode, Fig. 3 represent bold guesses at the possible evolution of the magnetic surfaces for this instability... If such reconnections were to take place in the doubletearing mode, it would be accompanied by a rapid redistribution of the current and electron heat along the new convoluted and extended magnetic surfaces.

An exact method for determining the threshold of reconnection for odd-period orbits was presented in Ref. [23]. This method uses the primitive function $S(x, y)$, which is a generalization of the generating function of canonical transformations (see, e.g. Ref.[25]). For the SNM, it is given by

$$
S(x, y)=-\frac{2 a}{3}[y-b \sin (2 \pi x)]^{3}+\frac{b}{2 \pi} \cos (2 \pi x) .
$$

The action of a periodic orbit $\left\{\boldsymbol{z}_{1}, \boldsymbol{z}_{2}, \ldots, \boldsymbol{z}_{n}\right\}$ is defined by

$$
A_{n}\left(\boldsymbol{z}_{1}, \boldsymbol{z}_{2}, \ldots, \boldsymbol{z}_{n}\right)=\sum_{i=1}^{n} S\left(\boldsymbol{z}_{i}\right) .
$$

In the case of the SNM, Petrisor[23] states that at the reconnection threshold for an odd-period hyperbolic orbit, the action of the orbit is zero. (This result is valid for any orbit for which the up and down orbits on a symmetry line have opposite symmetry types, which is the case for odd-period orbits in maps with quadratic twist.)

We have implemented this idea numerically. The main idea is to conduct a root search for the zeros of the action, considered as a function of the perturbation parameter $b$, for any given value of $a$. By this method, we have obtained reconnection curves $b=\Psi(a)$ for a few low-period orbits. These are shown in Fig. 7.

\subsection{Meandering invariant tori}

In this subsection, we will focus on two aspects of meandering tori: their implications for transport and their nested structure.

Meander transport and global meanders The reconnection process outlined for odd-period orbits also takes place for the fixed points of the SNM. The only difference is that the location of the fixed points with winding number $0 / 1$ is independent of the parameters $(a, b)$, i.e., for all $a>0$ the fixed points are located at $(x, y)=(0, \pm 1)$ and $(1 / 2, \pm 1)$. Thus even though the manifolds of the hyperbolic fixed points reconnect, the points do not collide with increased perturbation. This implies that for all $b>\Psi_{0 / 1}(a)$, there are meandering tori. These we call "global meanders" because they change the topology of the phase space globally and not just locally as is the case for meanders around other periodic orbits. An example of such meanders is shown in Fig. 4.

We also note that the global meanders can traverse large regions in phase space. For example, for $(a, b)=$ $(0.001,2.1)$, Fig. 4 shows meanders that range from $y \approx$ -13 to $y \approx 5$. Their appearance can be understood by noting that the map is "integrable" for any nonzero value of $b$ if $a=0$. In this integrable case, there are no invariant tori that encircle the cylinder. However, the orbit of any point $\left(x_{0}, y_{0}\right)$ is parallel to $y$-axis:

$$
y_{n}=y_{0}+n b \sin \left(2 \pi x_{0}\right), \quad x_{n}=x_{0} .
$$

The orbits near $x=0$ and $x=1 / 2$ undergo small displacements in $y$ under iteration, while those close to $x= \pm 1 / 4$ are displaced in $y$ in increments of the order of $\sim b$. When

\footnotetext{
${ }^{3}$ As shown in Ref. [18] this is not the case for odd-period orbits.
} 

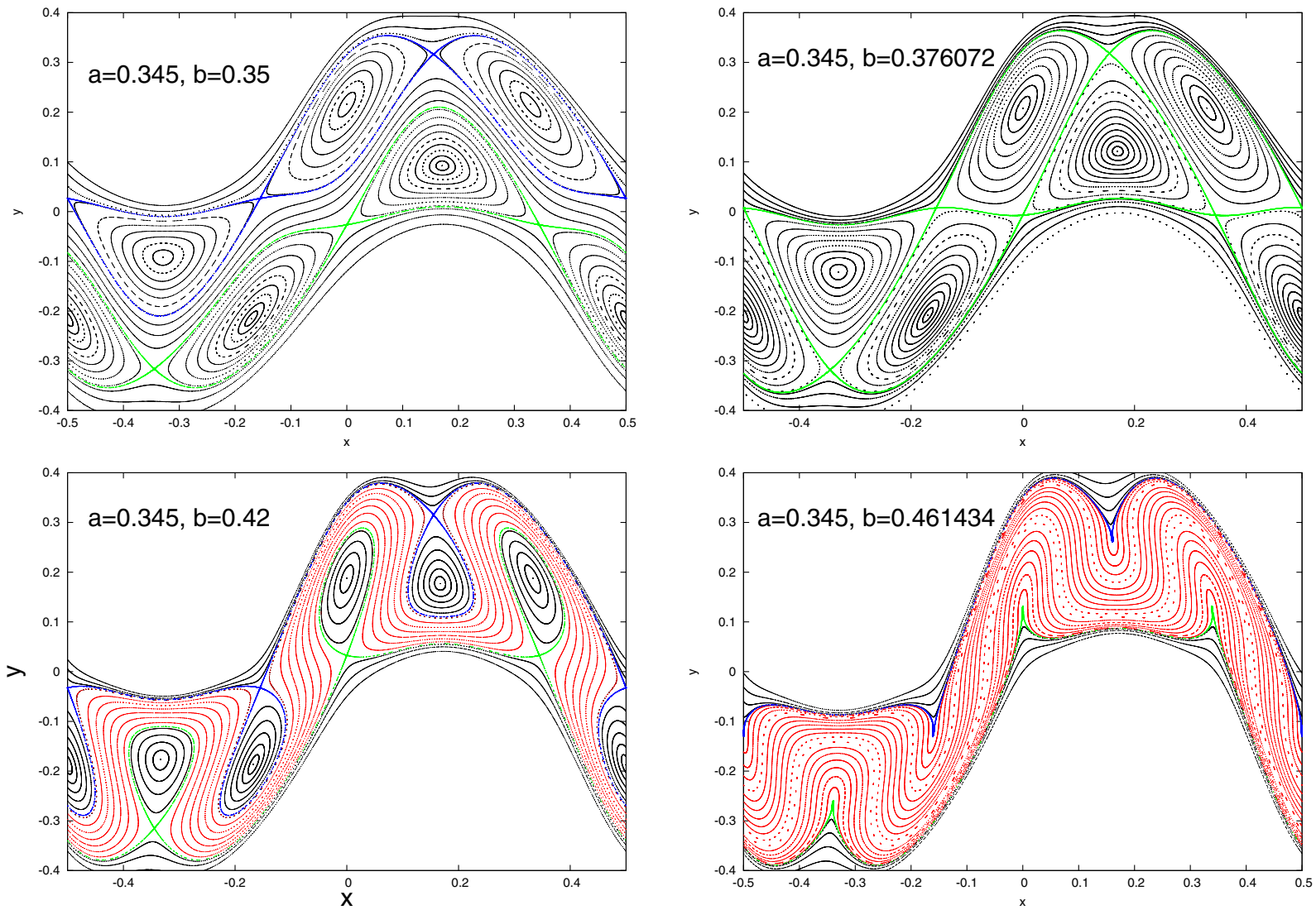

Figure 3. Reconnection of hyperbolic manifolds of up and down periodic orbits of odd period. The winding number for the chain shown here is $1 / 3$ and $a=0.345$. Meandering curves, shown in red, appear after reconnection. The orbits no longer exist after their collision [Fig. 3].

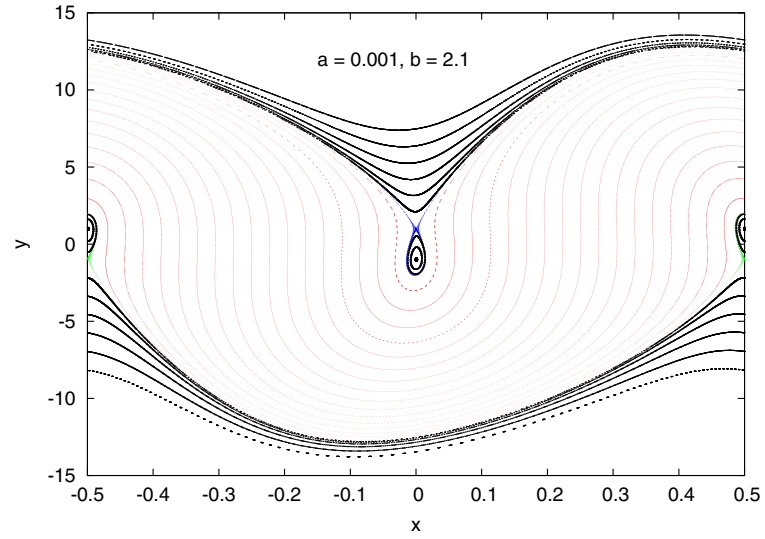

Figure 4. Meandering curves after reconnection of the fixed point with winding number $0 / 1$. Note the large range in $y$ and the existence of smooth tori even for the large perturbation $b=2.1$.

a small "perturbation" $a \neq 0$ is added, the orbits are deformed and the fixed points at $(0, \pm 1)$ and $(1 / 2, \pm 1)$ appear. The orbits still cover a large range in $y$, especially close to $y=0, x= \pm 1 / 4$, giving rise to phase space structures like those shown in Fig. 4. In the presence of such global meandering tori, transport in phase space can occur along these tori even when the tori are not broken. The implications of such transport scenarios in applications such as the structure of magnetic field lines are being investigated.

Nested meandering tori Another important aspect of meanders is their winding number. Fig. 3a-b shows a plot of the winding number as a function of the $y$-coordinate along the $s_{1}$ symmetry line at fixed parameter values, and Fig. 3a-b shows the corresponding phase space plots. The winding number increases monotonically until the hyperbolic point is reached, and then stays constant at 1/3 throughout the island. It starts decreasing in the meandering region, attaining a minimum at the shearless curve, and then increases again until the upper separatrix with winding number $1 / 3$ is reached. The latter cannot be seen in the plot because of insufficient resolution.

The meandering region itself has periodic orbits that can undergo reconnection and collision. Thus, if the perturbation is increased so that the minimum in Fig. 3a-b reaches a rational number, one can see the reconnection process occur locally inside the meandering region. A local maximum at the bottom of the minimum of the winding number plot will be an indicator of this "second order" reconnection, giving 
rise to "nested meanders." This is illustrated in Figs.3-a, 3-b for the reconnection of 18/19-period orbits inside the meandering region of the $1 / 1$-periodic orbit.

Thus we can imagine that the reconnection of periodic orbits inside this second order meandering region will lead to third order meanders and so on.[13] If this process continues, then at certain "critical" parameter values $b_{c}$, the limiting curve will have structure at all scales even though it is not at the point of breakup. Even apart from the structure of such a higher order meander, it is still an open question whether the last curve to break is a shearless meander or a non-meandering curve. As far as we know, there have been no studies of breakup of meandering curves using Greene's residue criterion.

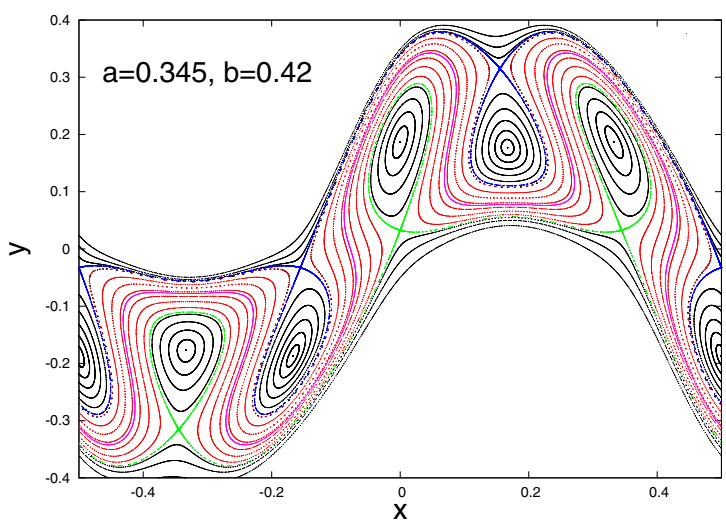

Figure 5a. Meandering curves after reconnection of 1/3-period orbit. The shearless curve is shown in purple.

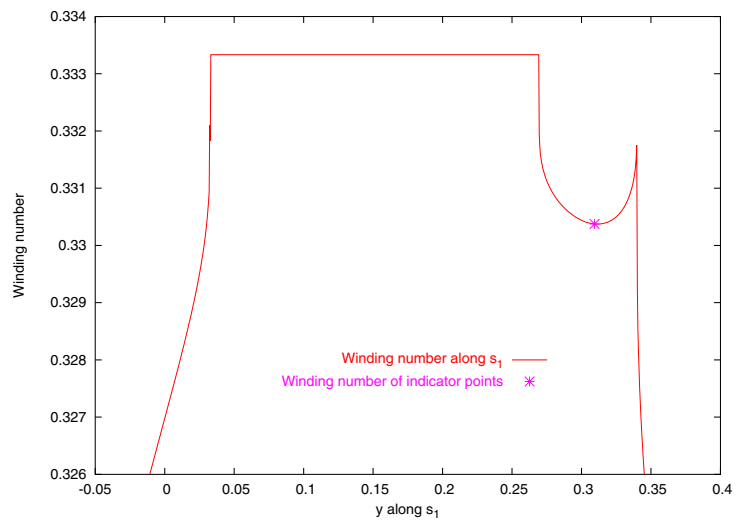

Figure $5 \mathrm{~b}$. The winding number along $s_{1}$. Note that the meandering region has winding number less than $1 / 3$. The winding number of the shearless torus is the local minimum as indicated. Note the "peak" on the right actually touches $1 / 3$. This is not seen here because of insufficient resolution.

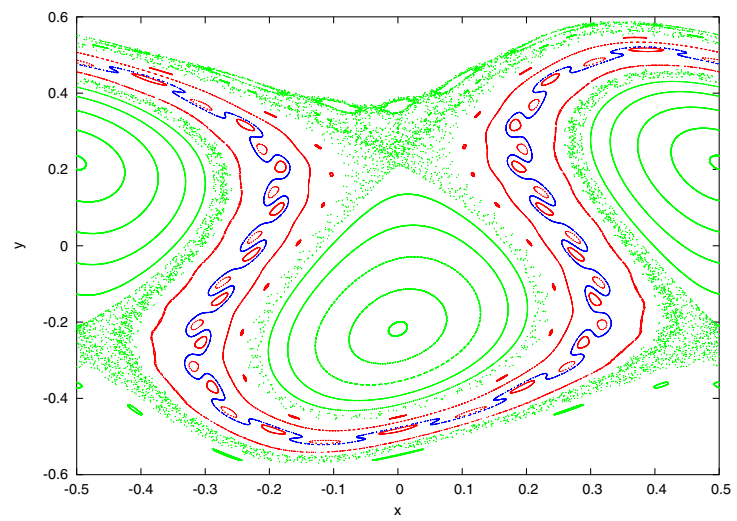

Figure 6a. Nested meanders around the 18/19 orbit inside the meandering region of $1 / 1$ orbit.

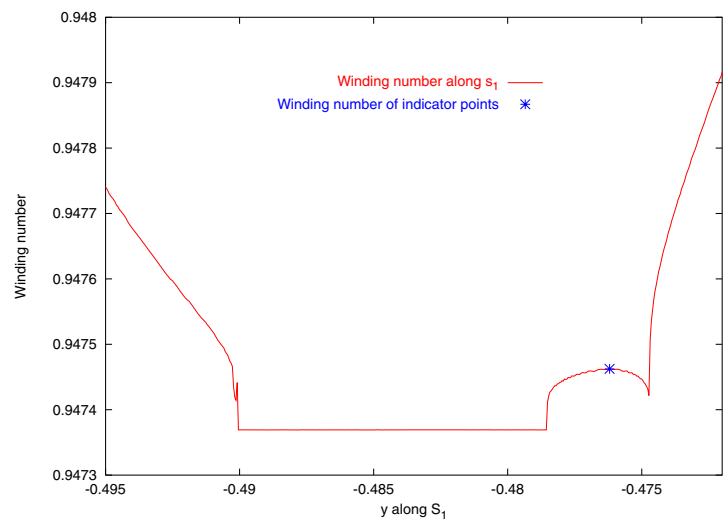

Figure $6 \mathrm{~b}$. The local maximum at the bottom of the local minimum, showing the presence of nested meanders. The "minimum" on the right should be at $18 / 19$, the same value as the plateau.

\section{Conclusion}

Another important use of the indicator points is to obtain a rough estimate of the threshold parameter values for breakup of the shearless torus.[18] This use is based on the observation that the indicator points belong to the shearless torus. For a given $(a, b)$, the indicator points are iterated many times (we used $10^{6}$ ). If the $y$ value stays below a threshold (we used $|y|<20$ ), it is assumed that the shearless curve exists and the point is plotted. Fig. 7 presents the results of using this procedure for the SNM.

This figure also depicts a few low-period bifurcation and reconnection curves. Note that this criterion provides an upper bound on the critical value for breakup because if the indicator point does not stay below the threshold, the shearless curve definitely has broken, but not vice-versa. The only exceptions are global meanders which reach past the bounds in $y$, but this happens only for large values of $b$ that are not shown in the figure.

There are a few important features to be noted about Fig. 7. 


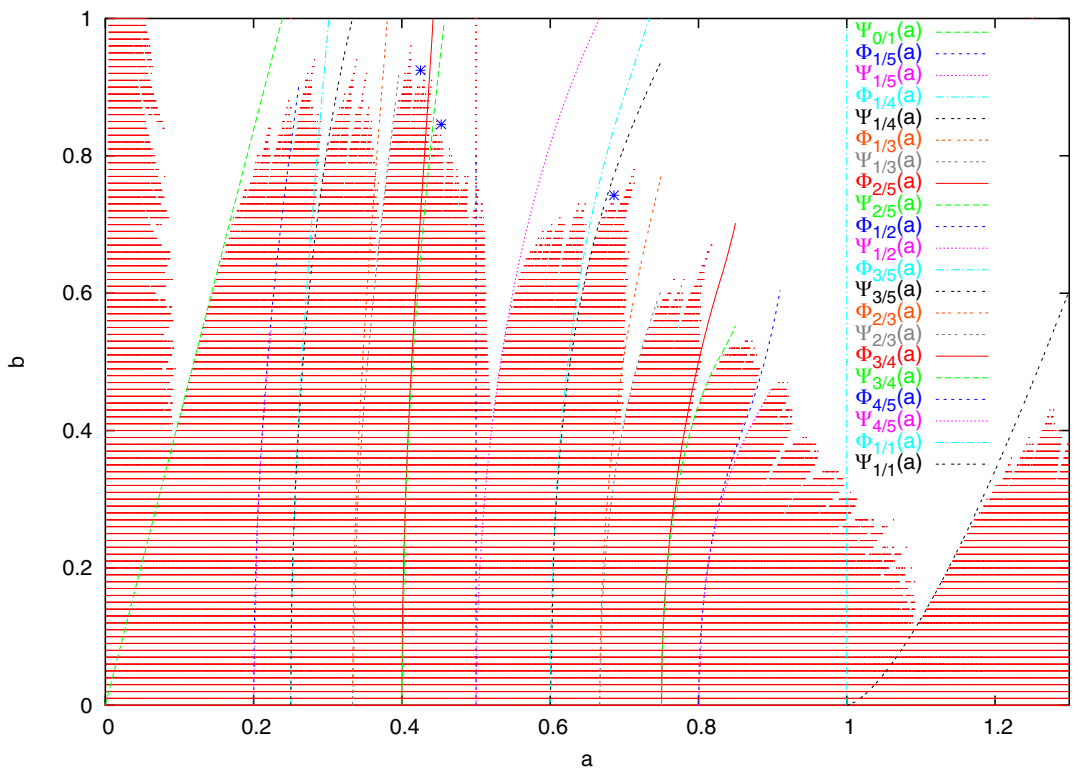

Figure 7. Parameter space showing the points for which shearless invariant tori exist. Also shown are the bifurcation and reconnection curves of low-period orbits and the critical points (marked by $*$ ) found using Greene's residue criterion.

- The $a=0.5$ line is the bifurcation curve of $1 / 2$ orbits on $s_{1}$. The indicator point $(a / 2+1 / 4,0)=$ $(0.5,0)$ is a period- 2 orbit and thus never crosses the $y$-threshold. Therefore the criterion fails for $a=0.5$.

- For $(a, b)$ values in the region left of the $0 / 1$ reconnection curve $\Psi_{0 / 1}(a)$ (i.e. the region $a \approx 0$ ), the map has global meanders. This region is transformed into the region near the $1 / 1$ reconnection curve (i.e. $a \approx 1$ ) under the reparametrization $a \rightarrow a+1$ and $b \rightarrow b \sqrt{a /(a+1)}$. Thus it might be easier to study the breakup of global meanders by studying the region in parameter space between $a=1$ and the $1 / 1$ reconnection curve.

- The plot generally shows a "dip" in the region between the reconnection and bifurcation curves. These are related to the presence of meanders, and further studies of breakup of meanders can reveal more clearly the structure in these "dips.”[17]

\section{Acknowledgments}

One of us (PJM) would like to thank the organizers for their hospitality at the joint X Latin American Workshop on Plasma Physics and the 7th Brazilian Meeting on Plasma Physics. This research was in part supported by U.S. Department of Energy Contract No. DE-FG01-96ER-54346 and by an appointment of A. Wurm to the U.S. Department of Energy Fusion Energy Postdoctoral Research Program administered by the Oak Ridge Institute for Science and Education.

\section{References}

[1] D. Del-Castillo-Negrete and P. J. Morrison, Phys. Fluids A 5, 948 (1993)

[2] E. Petrisor, Int. J. of Bifur. and Chaos 11, 497 (2001).

[3] R. Balescu, Phys. Rev. E 58, 3781 (1998).

[4] W. Horton, H. B. Park, J. M. Kwon, D. Strozzi, P. J. Morrison, and D. I. Choi, Phys. Plasmas 5, 3910 (1998).

[5] G. A. Oda and I. L. Caldas, Chaos, Solit. and Fract. 5, 15 (1995).

[6] T. H. Stix, Phys. Rev. Lett. 36, 10 (1976).

[7] K. Ullmann and I. L. Caldas, Chaos, Solit. and Fract. 11, 2129 (2000).

[8] M. Davidson, R. Dewar, H. Gardner, and J. Howard, Aust. J. Phys. 48, 871 (1995).

[9] T. Hayashi, T. Sato, H. J. Gardner, and J. D. Meiss, Phys. Plasmas 2, 752 (1995).

[10] H. R. Dullin, J. D. Meiss, and D. Sterling, Nonlinearity 13, 203 (2000).

[11] A. Delshams and R. de la Llave, SIAM J. Math. Anal. 31, 1235 (2000).

[12] J. Franks and P. Le Calvez, (1999), math.DS/9910152.

[13] C. Simó, Regul. Chaotic Dyn. 3, 180 (1998).

[14] D. Del-Castillo-Negrete, J. M. Greene, and P. J. Morrison, Physica D 91, 1 (1996).

[15] J. E. Howard and S. M. Hohs, Phys. Rev. A 29, 418 (1984).

[16] J. E. Howard and J. Humphreys, Physica D 80, 256 (1995).

[17] A. Wurm, A. Apte, and P. Morrison, in preparation.

[18] S. Shinohara and Y. Aizawa, Progr. of Theor. Phys. 100, 219 (1998).

[19] S. Shinohara and Y. Aizawa, Progr. of Theor. Phys. 97, 379 (1997). 
[20] A. Apte, A. Wurm, and P. J. Morrison, Chaos 13, 421 (2003).

[21] A. Apte, A. Wurm, and P. J. Morrison, Renormalization for breakup of invariant tori, Submitted to Physica D (2003).

[22] J. S. W. Lamb, J. Phys. A 25, 925 (1992).

[23] E. Petrisor, Chaos, Solit. and Fract. 14, 117 (2002).
[24] J. P. van der Weele, T. P. Valkering, H. W. Capel, and T. Post, Physica A 153, 283 (1988).

[25] Á. Haro, The primitive function of an exact symplectomorphism, Ph.D. thesis, Universitat de Barcelona (1998). 